\title{
Evidence for a bone-kidney axis regulating phosphate homeostasis
}

\section{Darryl Quarles}

Department of Medicine, Duke University Medical Center, Durham, North Carolina, USA

A novel circulation phosphaturic hormone is postulated to regulate systemic phosphate homeostasis. Two new studies (see the related articles beginning on pages 683 and 785 ) reveal that the phosphaturic factor FGF-23 is increased in hypophosphatemic subjects with McCuneAlbright syndrome and that secreted frizzled-related protein-4 (sFRP-4), a factor produced by tumors derived from subjects with tumor-induced osteomalacia, also has phosphaturic activity. It remains to be established whether FGF-23 and sFRP-4 represent two distinct phosphatonins or are somehow integrated in a novel phosphate-regulating bone-kidney axis.

J. Clin. Invest. 112:642-646 (2003). doi:10.1172/JCI200319687.

One must be aware of the tendency of the buman mind to distort stimuli to fit preconceived ideas. One tends to perceive what one wants and needs to perceive. One alters one's perceptions when one needs or wants to do so. - Louis E. Armstrong, Director, Indian Springs School, 1952-1972

\section{Evidence for multiple hormonal systems controlling phosphorus homeostasis}

The control of systemic phosphate homeostasis is not completely understood. Increased circulating levels of the calcemic parathyroid hormone (PTH) results in phosphaturia due to inhibition of sodium-dependent phosphate transport in the renal proximal tubule (1). Reductions in serum phosphate

Address correspondence to: L. Darryl Quarles, Department of Medicine, PO Box 3036, Duke University Medical Center, Durham, North Carolina 27710, USA. Phone: (919) 660-6853; Fax: (919) 684-4476; E-mail: quarl001@mc.duke.edu.

Conflict of interest: The author has declared that no conflict of interest exists.

Nonstandard abbreviations used: parathyroid hormone (PTH); autosomal dominant hypophosphatemic rickets (ADHR); X-linked hypophosphatemic rickets (XLH); McCune-Albright syndrome (MAS); tumor-induced osteomalacia (TIO); phosphate-regulating endopeptidase (PHEX); secreted frizzled-related protein-4 (sFRP-4); LDL receptor-related protein (LRP). concentrations stimulate renal proximal tubule production of $1,25(\mathrm{OH})_{2} \mathrm{D}_{3}$, which in turn increases the gastrointestinal absorption of calcium and phosphorus (2). The resulting increase in calcium leads to the secondary suppression of parathyroid hormone (PTH) and increases in sodium-dependent phosphate transport in the renal proximal tubule. The $\mathrm{PTH}$-vitamin $\mathrm{D}$ axis, however, is not sufficient to explain the physiological complexity of systemic phosphate homeostasis. Indeed, phosphate loading has a marked effect to reduce the net rate of proximal tubule phosphate reabsorption that is independent of PTH and vitamin $\mathrm{D}(3,4)$. In addition, a novel circulating phosphaturic factor, called phosphatonin, is postulated to be primarily responsible for modulating urinary phosphate excretion in a variety of hypophosphatemic disorders.

\section{Will the real phosphatonin please stand up?}

Evidence for the existence of phosphatonin comes from the study of the inherited hypophosphatemic disorders autosomal dominant hypophosphatemic rickets (ADHR) (5), X-linked hypophosphatemia (XLH) (6), and McCune-Albright syndrome (MAS) (7, 8 ), as well as the acquired hypophosphatemic disorder tumor-induced osteomalacia (TIO; also called oncogenic osteomalacia, or $\mathrm{OOH}$ ) (9-11). $\mathrm{X}$-linked hypophosphatemic rickets (XLH) and antosomal dominant hypophosphatemic rickets (ADHR) characterized by hypophosphatemia due to impaired renal tubular reabsorption of phosphate, inappropriately normal or decreased $1,25(\mathrm{OH})_{2} \mathrm{D}_{3}$ production, and defective calcification of cartilage and bone. TIO is a tumormediated hypophosphatemic disorder with phenotypic features similar to those of the hereditary phosphate wasting disorders. MAS is a hereditary fibrous bone dysplasia caused by activating mutations of $\mathrm{G}_{\mathrm{s}} \alpha$ that is sometimes associated with hypophosphatemic rickets. Based on the shared phenotype of these disorders, phosphatonin is predicted to be a circulating protein that inhibits sodiumdependent phosphate reabsorption by the renal proximal tubule through mechanisms distinct from $\mathrm{PTH}$, impairs mineralization of bone and cartilage to a degree greater than expected from the accompanying hypophosphatemia, and counters hypophosphatemia-mediated increases in the renal production of $1,25(\mathrm{OH})_{2} \mathrm{D}_{3}$. These disorders may share a common pathogenesis mediated by a single phosphatonin or represent distinct entities with common features caused by different phosphatonins. At present there are at least three factors with the predicted characteristics of phosphatonin.

\section{FGF-23}

FGF-23 appears to have all of the predicted biological properties of phosphatonin. FGF-23 is an approximately $26-\mathrm{kDa}$ circulating protein consisting of an N-terminal FGF homology domain and a novel 71-amino acid Cterminus of uncertain function. In vivo studies establish that full-length FGF-23 is a phosphaturic hormone (12-15). In addition, FGF-23-induced phosphaturia does not lead to upregulation of $1,25(\mathrm{OH})_{2} \mathrm{D}_{3}$ production as occurs with $\mathrm{PTH}$-induced phosphaturia. Treatment with FGF-23 causes a more severe form of rickets and osteo- 


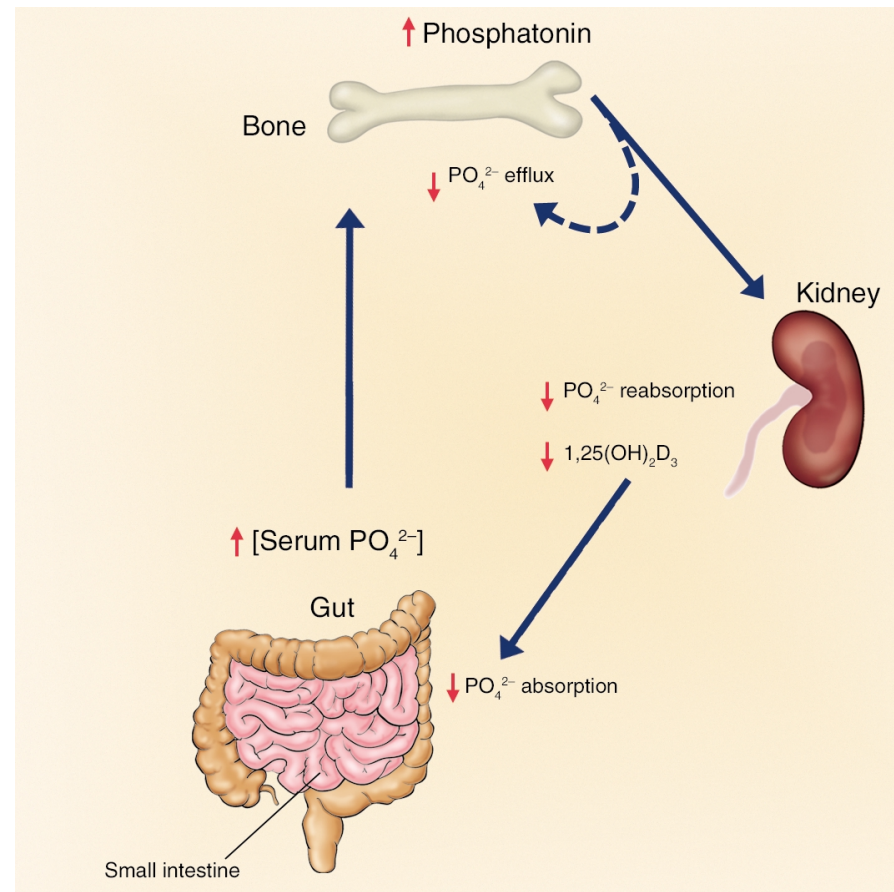

Figure 1

A bone-kidney axis regulating renal phosphate handling and skeletal mineralization. A phosphaturic hormone, phosphatonin, stimulates renal phosphate excretion. Osteoblasts in bone may be the source of phosphatonin. Increased release of phosphatonin leads to autocrine effects to regulate bone mineralization of ECM and systemic effects to cause phosphaturia.

malacia than is observed with hypophosphatemia alone, suggesting a direct effect of FGF-23 on cartilage and bone. Conversely, preliminary characterization of FGF-23-null mice shows increments in serum phosphate and increased $1,25(\mathrm{OH})_{2} \mathrm{D}_{3}$ production, thereby confirming the essential and nonredundant role of FGF-23 in regulating phosphate homeostasis through PTH-independent mechanisms (16). Cleavage of FGF-23 at the 176-RXXR-179 motif by a furin-type convertase sensitive to inhibition by decanoyl-Arg-Val-Lys-Arg-chloromethyl-ketone (17) is reported to generate biologically inactive $\mathrm{N}$ - and $\mathrm{C}$ terminal fragments (14).

There is also an association between increments in FGF-23 and hypophosphatemic disorders. ADHR is caused by missense mutations in FGF-23 at the 176-RXXR-179 motif that prevent the hydrolysis and inactivation of the full-length bioactive FGF-23 (13). The $\mathrm{X}$-linked dominant hypophosphatic disorder $\mathrm{XLH}$, which is caused by inactivating mutations of the cell surface metalloprotease phosphate-regulating endopeptidase (PHEX) $(6,18)$, also is characterized by increased circulating levels of FGF-23 that correlate with the degree of hyposphosphatemia (19). Although initial studies suggested that FGF-23 might be a substrate for PHEX (20), additional studies have been unable to confirm this (17). Rather, quantitative real-time RT-PCR analysis of bone from the Hyp mouse homologue of XLH identifies significant increases in the expression of FGF-23 in bone as well as in Hypderived osteoblast cultures (17). Thus, increased FGF-23 production by bone may be the cause of increased circulating levels of FGF-23 in XLH, rather than decreased degradation. Regardless, additional intermediate steps involving PHEX substrates and/or interacting proteins are necessary to link inactivating PHEX mutations to increased FGF-23 production. FGF-23 has also been identified in the tumors of patients with TIO (9), and circulating levels of FGF-23 are increased in subjects with TIO $(19,21,22)$.

In this issue of the JCI, Riminucci et al. have provided evidence that FGF-23 is implicated in the pathogenesis of phosphaturia in MAS (23). MAS, which is caused by activating mutations of $\mathrm{G}_{\mathrm{s}} \alpha$ that lead to fibrous dysplastic lesions of bone, is often associated with impaired skeletal mineralization and humoral-induced phosphaturia $(7,8)$. The finding that circulating levels of FGF-23 are also increased in MAS is consistent with the notion that all of these phosphaturic disorders have a common pathogenesis mediated by elevated FGF-23. More importantly, the finding that FGF-23 is produced by normal and fibrous dysplasia osteoprogenitors and bone-forming cells in vivo and in vitro implicates bone and osteoblasts as the tissue/cell type that produces FGF-23, similar to findings of increased $f g f 23$ expression in $H y p$ bone associated with inactivating Phex mutations. At present, FGF-23 is the leading candidate for phosphatonin.

\section{MEPE}

Differential gene-expression profiling of TIO tumors by a variety of techniques has also identified the matrix extracellular phosphoglycoprotein $\operatorname{MEPE}(9,13)$, also named OF45 (24). MEPE was isolated and cloned from a TIO tumor cDNA library (11) and independently isolated and cloned from the rat and mouse based on its ability to regulate mineralization (25). MEPE belongs to the small integrin-binding ligand $\mathrm{N}$-linked glycoprotein (SIBLING) family of proteins. The SIBLING family of ECM proteins are involved in regulating the mineralization of ECM and include bone sialoprotein, osteopontin, dentin sialophosphoprotein, and dentin matrix protein-1 (26). MEPE-null mice exhibit increased osteoblast-mediated mineralization, indicating that MEPE plays an inhibitory role in bone formation in mice (24). It has been postulated that MEPE may have phosphaturic actions (10); however, preliminary studies indicate that transfer of MEPE deficiency onto the Hyp mouse background fails to rescue the hypophosphatemia in $H y p$ mice, suggesting that MEPE is not the cause of phosphaturia in the setting of inactivating Phex mutations (27). Other studies suggest that Phex may modify the hydrolysis of MEPE (28). The biological significance of the later finding is uncertain, but it might implicate MEPE in the local regulation of mineralization through Phex-dependent mechanisms. 
Secreted frizzled-related protein-4

Secreted frizzled-related protein-4 (sFRP-4) was also identified in serial analysis of gene expression of TIO (9). In this issue of the JCI, Berndt et al. have extended these observations by showing that sFRP-4 is present in normal human serum and is increased in the serum in TIO (29).

sFRP-4 is an extracellular antagonist of Wnt signaling. Wnt's interact with a receptor complex containing a member of the frizzled family of heptahelical receptors and $L D L$ receptor-related proteins (LRPs), which includes LRP-5 and LRP-6. The binding and sequestration of Wnt's by sFRPs prevents Wnt-dependent activation of the frizzled/LRP receptor complex. Given the primary importance of Wnt pathways in the regulation of proliferation, differentiation, and apoptosis and the finding that sFRPs are expressed by nonphosphaturic tumors, it is diffi- cult to conceive how sFRP-4 could selectively target the renal proximal tubule to inhibit sodium-dependent phosphate uptake. Indeed, there is no known precedent for Wnt-dependent regulation of phosphate homeostasis or for hormonal actions of sFRPs. Rather, sFRPs are more typically found at the plasma membrane and/or in the ECM. Thus, the initial bias is that sFRP-4 is an unlikely candidate for phosphatonins.

However, the study by Berndt et al. (29) requires that we reevaluate this preconception and consider the possible role of sFRP-4 in the pathogenesis of phosphaturia in TIO and other hypophosphatemic disorders. In this regard, the authors provide compelling evidence that sFRP-4 has characteristics of phosphatonin. Recombinant sFRP-4 inhibits sodium-dependent phosphate transport in cultured opossum renal epithelial cells, indicating a possible direct action of sFRP-4 on proximal tubular phosphate transport. In addition, the systemic administration of recombinant sFRP-4 caused phosphaturia in normal rats without stimulating $1 \alpha$-hydroxylase activity in the kidney. The inhibition of renal phosphate reabsorption was associated with evidence for sFRP-4 antagonizing Wnt-dependent $\beta$-catenin pathways in the kidney. These observations support the existence of more than one phosphatonin (30).

\section{A bone-kidney axis regulating phosphate homeostasis and mineralization}

How do we reconcile the observations that FGF-23 and sFRP-4 are both phosphatonins with the hypothesis that hereditary and acquired hypophosphatemic disorders share a common pathogenesis? One possibility is that these factors are integrated at the level

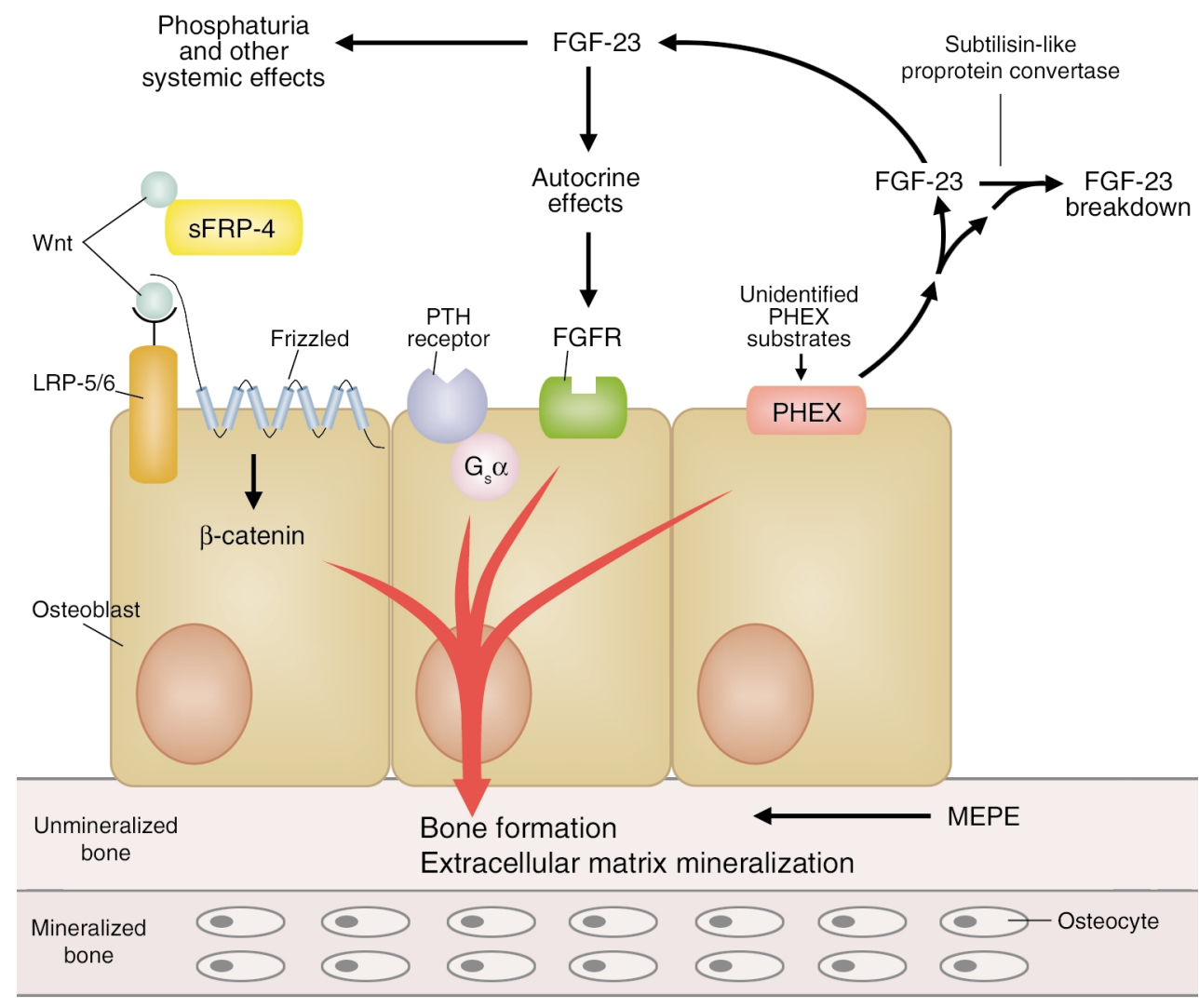

\section{Figure 2}

Osteoblasts express components necessary for coordinating mineralization of ECM and phosphate handling by the kidney. FGF-23 secreted from osteoblasts under the control of PHEX and possibly $\mathrm{G}_{\mathrm{s}} \alpha$-dependent pathways has systemic actions to regulate renal phosphate handling as well as potential autocrine effects to regulate osteoblast-mediated bone mineralization. The LRP-Wnt signaling pathway in osteoblasts regulates bone mass and, in conjunction with PHEX and FGF-23, may also participate in coordinating renal phosphate conservation to meet the needs of osteoblast-mediated mineralization. 
of the osteoblast into a novel phosphate-regulating hormonal axis that controls the renal tubular reabsorption of phosphate and mineralization (Figures 1 and 2).

Several compelling observations support the central role of bone in coordinating renal phosphate handling to meet the needs for mineralization. First, the association between increased FGF-23 transcripts in bone, increased circulating FGF-23, and hypophosphatemia provides a strong case for a possible endocrine function of the skeleton to inhibit sodium-dependent phosphate reabsorption by the renal proximal tubule through the production and secretion of FGF-23 (Figure 1). Indeed, osteoblasts derived from $H y p$ mice produce a factor capable of inhibiting sodium-dependent phosphate transport in renal tubular cells (31), implicating bone as a possible source of phosphatonin. The current findings by Riminucci et al. (23) and recent studies in Hyp mice (17) indicate that osteoblasts are a source of FGF-23 in bone. Second, there is evidence for an intrinsic defect in osteoblast-mediated mineralization. In this regard, the severity of rickets and osteomalacia in XLH, ADHR, and TIO appears to be greater than can be explained by hypophosphatemia. Hypophosphatemia in mice lacking the renal proximal tubule sodium-dependent phosphate transporter Npt2 does not cause rickets and osteomalacia as severe as in the hereditary hypophosphatemic disorders (32). Hypderived osteoblasts lacking a functional Phex also secrete a putative factor, referred to as minhibin (33), which inhibits mineralization independent of hypophosphatemia.

The severe bone phenotype and apparent intrinsic abnormalities of osteoblast-mediated mineralization in Hyp mice suggest local regulation of the mineralization process. This might occur via autocrine effects of FGF-23 to regulate skeletal mineralization and/or actions of Phex on the expression and/or metabolism of ECM proteins involved in mineralization (Figure 2). There is indirect evidence for both mechanisms in that FGF receptors are present in osteoblasts and chondrocytes that might be targets of FGF-23 actions. Increased mepe transcripts in bone of
Hyp mice are consistent with involvement of this ECM protein in mineralization. The further observation that increments in mepe are highly correlated with the level of $f g f 23$ expression in Hyp bone (17) suggests that FGF-23 may have an autocrine effect to increase MEPE production by osteoblasts. The current investigation by Riminucci et al. (23), showing an association between activation of $\mathrm{G}_{\mathrm{s}} \alpha$ and FGF-23 production, also opens the possibility of PTH stimulation of FGF-23 by osteoblasts, thereby linking FGF-23 to the PTHvitamin $D$ axis.

Could sFRP-4 also indirectly affect renal phosphate transport and mineralization through a primary skeletal effect? This possibility is suggested by the importance of osteoblastic Wnt signaling in the control of bone mass. In this regard, a Gly171Val mutation in LRP-5 results in autosomal dominant high bone density due to augmented osteoblast-mediated bone formation $(34,35)$, whereas disruption of LRP5 leads to a decrease in osteoblast proliferation and low bone mass in mice (36). Based on these observations, sFRP-4 inhibition of Wnt-dependent bone formation might secondarily reduce the need for renal phosphate conservation. It would be of interest to determine whether sFRP-4 is regulated in response to stimuli that lead to alterations in bone mass. It also would be of interest to determine whether Wnt-dependent signaling affects PHEX activity, particularly since the C-terminal half of sFRPs contains a motif found in tissue inhibitors of metalloproteases. In addition, the effects of sFRP-4 and Wnt on osteoblast-derived phosphaturic and mineralization-inhibiting factors need to be investigated.

Regardless, a bone-kidney hormonal axis would provide a mechanism for the skeleton to communicate with the kidney to coordinate the mineralization of ECM with the renal handling of phosphate. Osteoblasts are well suited for coordinating systemic phosphate homeostasis and mineralization, since they express all of the implicated components of a possible bone-kidney axis, including PHEX, FGF-23, MEPE, and LRP-5/Wnt, as well as frizzled, FGF, and PTH receptors (Figure 2). In addition, autocrine effects of phosphatonin on osteoblasts could regulate the production of ECM proteins that regulate mineralization. Proving the existence of this bone-kidney axis and defining its physiological role will require additional investigations. The available data, however, are not inconsistent with the possibility that FGF-23 produced by osteoblasts has phosphaturic actions on the kidney and autocrine effects on osteoblasts to modulate the mineralization of bone (Figure 1).

\section{Acknowledgments}

This work was supported in part by grant RO1-AR45955 from the National Institute of Arthritis and Musculoskeletal and Skin Diseases. I thank Cristy McGranahan for secretarial assistance in the preparation of this manuscript.

1. Murer, H., et al. 1999. Posttranscriptional regulation of the proximal tubule $\mathrm{NaPi}$-II transporter in response to $\mathrm{PTH}$ and dietary $\mathrm{P}(\mathrm{i})$. Am. J. Physiol. 277:F676-F684.

2. Portale, A.A., Halloran, B.P., and Morris, R.C., Jr. 1989. Physiologic regulation of the serum concentration of 1,25-dihydroxyvitamin D by phosphorus in normal men. J. Clin. Invest. 83:1494-1499.

3. Trohler, U., Bonjour, J.P., and Fleisch, H. 1976 Inorganic phosphate homeostasis. Renal adaptation to the dietary intake in intact and thyroparathyroidectomized rats. J. Clin. Invest. 57:264-273.

4. Brautbar, N., Walling, M.W., and Coburn, J.W 1979. Interactions between vitamin D deficiency and phosphorus depletion in the rat. J. Clin. Invest. 63:335-341.

5. White, K.E., et al. 2000. Autosomal dominant hypophosphataemic rickets is associated with mutations in FGF23. Nat. Genet. 26:345-348.

6.1995. A gene (PHEX) with homologies to endopeptidases is mutated in patients with $\mathrm{X}$ linked hypophosphatemic rickets. The HYP Consortium. Nat. Genet. 11:130-136.

7. Collins, M.T., et al. 2001. Renal phosphate wasting in fibrous dysplasia of bone is part of a generalized renal tubular dysfunction similar to that seen in tumor-induced osteomalacia. J. Bone Miner. Res. 5:806-813.

8. Yamamoto, T., et al. 2001. Hypophosphatemic rickets accompanying McCune-Albright syn drome: evidence that ahumoral factor causes hypophosphatemia. J. Bone Miner. Metab. 5:287-295.

9. De Beur, S.M., et al. 2002. Tumors associated with oncogenic osteomalacia express genes important in bone and mineral metabolism. J. Bone Miner. Res. 17:1102-1110.

10. Quarles, L.D., and Drezner, M.K. 2001. Pathophysiology of X-linked hypophosphatemia tumor-induced osteomalacia, and autosomal dominant hypophosphatemia: a perPHEXing problem. J. Clin. Endocrinol. Metab. 86:494-496.

11. Rowe, P.S., et al. 2000. MEPE, a new gene expressed in bone marrow and tumors causing osteomalacia. Genomics. 67:54-68.

12. Saito, H., et al. 2003. Human fibroblast growth factor-23 mutants suppress $\mathrm{Na}+$-dependent phosphate co-transport activity and 1alpha,25dihydroxyvitamin D3 production. J. Biol. Chem. 278:2206-2211.

13. Shimada, T., et al. 2001. Cloning and characteri- 
zation of FGF23 as a causative factor of tumorinduced osteomalacia. Proc. Natl. Acad. Sci.U.S. A. 98:6500-6505.

14. Shimada, T., et al. 2002. Mutant FGF-23 responsible for autosomal dominant hypophosphatemic rickets is resistant to proteolytic cleavage and causes hypophosphatemia in vivo. Endocrinology. 143:3179-3182.

15. Yamashita, T., Konishi, M., Miyake, A., Inui, K., and Itoh, N. 2002. Fibroblast growth factor (FGF)-23 inhibits renal phosphate reabsorption by activation of the mitogen-activated protein kinase pathway. J. Biol. Chem. 277:28265-28270.

16. Shimada, T., et al. 2002. Targeted ablation of FGF-23 causes hyperphosphatemia, increased 1,25-dyidrxyvitam D levels and severe growth retardation. J. Bone Miner. Res. 17:S168. (Abstr.)

17. Liu, S., et al. 2003. Regulation of FGF23 expression but not degradation by Phex. J. Biol. Chem. 10.1074/jbc.M304544200.

18. Holm, I.A., Huang, X., and Kunkel, L.M. 1997. Mutational analysis of the PHEX gene in patients with X-linked hypophosphatemic rickets. Am. J. Hum. Genet. 60:790-797.

19. Weber, T.J., Liu, S., Indirdason, O.S., and Quarles, L.D. 2003. Serum FGF23 levels in normal and disordered phosphorus homeostasis. J. Bone Miner. Res. 18:1227-1234.

20. Bowe, A.E., et al. 2001. FGF-23 inhibits renal tubular phosphate transport and is a PHEX substrate. Biochem. Biophys. Res. Commun. 284:977-981.

21. Jonsson, K.B., et al. 2003. Fibroblast growth factor 23 in oncogenic osteomalacia and X-linked hypophosphatemia. N. Engl.J. Med. 348:1656-1663

22. Yamazaki, Y., et al. 2002. Increased circulatory level of biologically active full-length FGF-23 inpatients with hypophosphatemic rickets/osteomalacia. J. Clin. Endocrinol. Metab. 87:4957-4960.

23. Riminucci, M., et al. 2003. FGF-23 in fibrous dysplasia of bone and its relationship to renal phosphate wasting. J. Clin. Invest. 112:683-692. doi:10.1172/JCI200318399.

24. Gowen, L.C., et al. 2003. Targeted disruption of the osteoblast/osteocyte factor 45 gene (OF45) results in increased bone formation and bone mass. J. Biol. Chem. 278:1998-2007.

25. Petersen, D.N., Tkalcevic, G.T., Mansolf, A.L., Rivera-Gonzalez, R., and Brown, T.A. 2000. Identification of osteoblast/osteocyte factor 45 (OF45), a bone-specific cDNA encoding an RGD-containing protein that is highly expressed in osteoblasts and osteocytes. J. Biol. Chem. 275:36172-36180.

26. Qin, C., et al. 2003. Evidence for the proteolytic processing of dentin matrix protein 1: identification and characterization of processed fragments and cleavage sites. J. Biol. Chem. 10.1074/jbc.M305315200.

27. Liu, S., Brown, T.A., Xiao, Z., Guo, R., and Quarles, L.D. 2003. Deletion of Mepe in Hyp mice fails to correct hypophosphatemia but partially rescues abnormal mineralization ex vivo. J. Bone Miner. Res. In press.

28. Guo, R., Rowe, P.S.N., Liu, S., and Quarles, L.D 2002. Inhibition of MEPE cleavage by Phex. Biochem. Biophys. Res. Commun. 297:38-45.
29. Berndt, T., et al. 2003. Secreted frizzled-related protein 4 is a potent tumor-derived phosphaturic agent. J. Clin. Invest. 112:785-794. doi:10.1172/ JCI200318563

30. Kumar, R. 2002. New insights into phosphate homeostasis: fibroblast growth factor 23 and frizzled-related protein- 4 are phosphaturic factors derived from tumors associated with osteomalacia. Curr. Opin. Nephrol. Hypertens. 11:547-553.

31. Nesbitt, T., et al. 1999. Coordinated maturational regulation of PHEX and renal phosphate transport inhibitory activity: evidence for the pathophysiological role of PHEX in X-linked hypophosphatemia. J. Bone Miner. Res. 14:2027-2035.

32. Beck, L., et al. 1998. Targeted inactivation of $\mathrm{Npt} 2$ in mice leads to severe renal phosphate wasting, hypercalciuria, and skeletal abnormalities. Proc. Natl. Acad. Sci. U. S. A. 95:5372-5377.

33. Xiao, Z.S., et al. 1998. Intrinsic mineralization defect in Hyp mouse osteoblasts. Am. J. Physiol. 275:E700-E708

34. Boyden, L.M., et al. 2002. High bone density due to a mutation in LDL-receptor-related protein 5. N. Engl. J. Med. 346:1513-1521.

35. Little, R.D., et al. 2002. A mutation in the LDL receptor-related protein 5 gene results in the autosomal dominant high-bone-mass trait. Am. J. Hum. Genet. 70:11-19.

36. Kato, M., et al. 2002. Cbfa1-independent decrease in osteoblast proliferation, osteopenia, and persistent embryonic eye vascularization in mice deficient in Lrp5, a Wnt coreceptor. J. Cell Biol. 157:303-314.

\title{
Come forth CD1d: Hsp110 in the regulation of intestinal epithelial CD1d expression
}

\author{
Christopher V. Nicchitta
}

Department of Cell Biology, Duke University Medical Center, Durham, North Carolina, USA

\begin{abstract}
CD1d, a nonclassical MHC class I-like molecule, is prominently expressed on intestinal epithelial cells and is thought to function in the regulation of intestinal intraepithelial lymphocyte activity. Hsp110, an abundant heat shock protein present in essentially all mammalian tissues, has now been shown to upregulate CD1d expression in colonic tissue culture cell lines (see the related article beginning on page 745). Might this abundant chaperone serve an autocrine function in the regulation of CD1d expression?
\end{abstract}

J. Clin. Invest. 112:646-648 (2003). doi:10.1172/JCI200319614.

Intestinal epithelial cells (IECs) serve as the cellular barrier between the enormously challenging antigenic

Address correspondence to: Christopher V.
Nicchitta, Department of Cell Biology,
Duke University Medical Center, Durham,
North Carolina 27710, USA.
Phone: (919) 684-8948; Fax: (919) 684-5481;
E-mail: c.nicchitta@cellbio.duke.edu.
Conflict of interest: The author has declared
that no conflict of interest exists.
Nonstandard abbreviations used: intestinal
epithelial cell (IEC); inflammatory bowel
disease (IBD); heat shock protein 110 (Hsp110). environment of the intestinal lumen consisting of dietary antigens, viruses, bacteria, and fungi - and the mucosal immune system, the largest lymphoid tissue of the body. Given this remarkable immunological challenge, it comes as no surprise that both normal and pathological intestinal epithelial physiology involves complex interactions among the immune and nonimmune cells of the gut, the intestinal flora, and the activities of the sympathetic, parasympathetic, and enteric nervous systems. Unfortunately, such complex interactions often go awry, resulting in the chronic, $\mathrm{T}$ lymphocyte-mediated mucosal injury that characterizes inflammatory bowel diseases (IBDs).

The molecular etiology of IBDs is under continuing investigation, with current views emphasizing interactions between environmental pathogens and an altered, or genetically permissive, immune system (1). Recent studies have implicated CD1d in the regulation of intestinal inflammation, though here again, the precise immunological mechanism for such regulation remains to be identified (2-4). CD1d is a nonclassical MHC molecule that functions in the development of NK1.1 $1^{+} \mathrm{T}$ cells and has been implicated in the process of IEC-elicited $\mathrm{T}$ cell proliferation $(5,6)$. Interestingly, antibody cross-linking of CD1d has been demonstrated to elicit epithelial IL-10 production (7). IL-10 is known to serve important functions in the regulation of mucosal inflammation, and, importantly, IL-10-deficient mice coincidentally develop IBD (reviewed in ref. 8). From these observations has come the intriguing suggestion that ligation of CD1d may serve to suppress mucosal inflamma- 